\title{
A NOTE ON APPROXIMATION PROPERTIES OF THE OSELEDETS SPLITTING
}

\author{
CHAO LIANG, GANG LIAO, AND WENXIANG SUN \\ (Communicated by Yingfei Yi)
}

\begin{abstract}
We prove that the Oseledets splitting, mean angle and independence number of an ergodic hyperbolic measure of a $C^{1+r}$ diffeomorphism can be approximated by those of atomic measures on hyperbolic periodic orbits. This removes the assumption on simple spectrum in an earlier paper by the authors and strengthens Katok's closing lemma by presenting more information about not only the state space but also its linearization.
\end{abstract}

\section{IntRoduCtion AND RESUlts}

In dynamical systems hyperbolicity is always accompanied by abundant periodic orbits, and thus the study on periodic approximation properties is an important topic. Among these are the approximation of Lyapunov exponents and approximation of an angle between Oseledets bundles. In [10, Wang and Sun proved that the Lyapunov exponents of hyperbolic measures can be approximated by those of nearby periodic orbits in the setting of nonuniformly hyperbolic systems, and in [1] Dai considered the approximation of Lyapunov exponents for linear cocycles. In [4], Liang, Liu and Sun studied more properties on periodic points: Oseledets splitting, mean angle, independence number and Liao style number. These approximation properties all together contribute more extensive information on the linearization of diffeomorphisms and thus strengthen Katok's closing lemma. However, the results in 4] are proved only for diffeomorphisms with simple spectrum due to the technique limitation: a given measure in a state manifold has only finitely many covering measures on the Oseledets frame bundle by Sun and Vargas 9 .

In the present note, we prove the general approximation properties on Oseledets splitting without the assumption of simple spectrum. To overcome the obstruction, we need a conceptually different approach. We consider the Oseledets splitting, rather than a column of vectors, as an element, construct the Oseledets flag but not the frame bundle, and prove the isolation of the covering measure on the flag

Received by the editors May 12, 2012, and, in revised form, November 1, 2012 and November 12, 2012.

2010 Mathematics Subject Classification. Primary 37D05, 37D25, 37C25.

Key words and phrases. Oseledets splitting, mean angle, independence number.

The first author was supported by NSFC (\#10901167), Beijing Higher Education Young Elite Teacher Project, and Program for Innovation Research in Central University of Finance and Economics.

The third author was supported by NSFC (\#11231001) and Doctoral Education Foundation of China. 
for a given measure on the state manifold. This uniqueness instead of the finiteness of covering measures in [4] allows the absence of the condition on simple spectrum in [4] for all the periodic approximation properties.

Let $M$ be a compact connected $d$-dimensional Riemannian manifold without boundary. For $1 \leq i \leq d, x \in M$, let $G(i, x)$ denote the Grassmann manifold formed by all $i$-dimensional linear spaces in $T_{x} M$. Given integers $n_{1}, n_{2}, \cdots, n_{s}$ with $1 \leq n_{i} \leq d$ and $\sum_{i=1}^{s} n_{i}=d$, we define a bundle

$$
\mathcal{U}\left(n_{1}, \cdots, n_{s}\right)=\bigcup_{x \in M} \mathcal{U}\left(n_{1}, \cdots, n_{s} ; x\right),
$$

where the fiber over $x$ is

$$
\mathcal{U}\left(n_{1}, \cdots, n_{s} ; x\right)=\left\{\left(E_{1}, \cdots, E_{s}\right) \in G\left(n_{1}, x\right) \times \cdots \times G\left(n_{s}, x\right)\right\} .
$$

Set $\mathcal{V}\left(n_{1}, \cdots, n_{s}\right)=\bigcup_{x \in M} \mathcal{V}\left(n_{1}, \cdots, n_{s} ; x\right)$, where the fiber over $x$ is

$$
\mathcal{V}\left(n_{1}, \cdots, n_{s} ; x\right)=\left\{\left(E_{1}, \cdots, E_{s}\right) \in \mathcal{U}\left(n_{1}, \cdots, n_{s} ; x\right) \mid E_{1} \oplus \cdots \oplus E_{s}=T_{x} M\right\} .
$$

It is immediate that by definition, $\mathcal{V}\left(n_{1}, \cdots, n_{s}\right)$ is open and

$$
\mathcal{U}\left(n_{1}, \cdots, n_{s}\right)=\overline{\mathcal{V}\left(n_{1}, \cdots, n_{s}\right)} .
$$

Given integers $1 \leq l_{1}<l_{2}<\cdots<l_{k}<d$, we define a flag bundle $\mathcal{W}\left(l_{1}, \cdots, l_{k}\right)=$ $\bigcup_{x \in M} \mathcal{W}\left(l_{1}, \cdots, l_{k} ; x\right)$, where the fiber over $x$ is

$$
\begin{aligned}
\mathcal{W}\left(l_{1}, \cdots, l_{k} ; x\right)=\left\{\left(F_{1}, \cdots, F_{k} ; H_{1}, \cdots, H_{k}\right) \mid\right. & F_{i} \in G\left(l_{i}, x\right), H_{i} \in G\left(d-l_{i}, x\right), \\
& \left.F_{i} \subset F_{i+1}, \quad H_{i+1} \subset H_{i}\right\} .
\end{aligned}
$$

Clearly, by the definition, $\mathcal{W}\left(l_{1}, \cdots, l_{k}\right)$ is compact.

Throughout this paper, we assume the following relations:

$$
k=s-1, \quad l_{i}=\sum_{j=1}^{i} n_{j}, \quad 1 \leq i \leq s-1 .
$$

For the sake of the statement, we write $\mathcal{U}=\mathcal{U}\left(n_{1}, \cdots, n_{s}\right), \mathcal{V}=\mathcal{V}\left(n_{1}, \cdots, n_{s}\right)$ and $\mathcal{W}=\mathcal{W}\left(l_{1}, \cdots, l_{k}\right)$. Given $\left(E_{1}, \cdots, E_{s}\right) \in \mathcal{V}$, we define

$$
\chi\left(\left(E_{1}, \cdots, E_{s}\right)\right)=\left(E_{1}, E_{1} \oplus E_{2}, \cdots, \bigoplus_{i=1}^{s-1} E_{i} ; \quad \bigoplus_{i=2}^{s} E_{i}, \cdots, E_{s-1} \oplus E_{s}, E_{s}\right) \in \mathcal{W} .
$$

Then $\chi: \mathcal{V} \rightarrow \chi(\mathcal{V})$ is a homeomorphism. Denote $\mathcal{W}_{1}=\chi(\mathcal{V})$ and $\chi^{-1}=\left.\chi^{-1}\right|_{\mathcal{W}_{1}}$.

For a $p$-frame $\xi=\left(u_{1}, u_{2}, \cdots, u_{p}\right)$ which spans a $p$-dimensional space $E$, we define $\operatorname{vol}(\xi)=\operatorname{vol}\left(u_{1}, u_{2}, \cdots, u_{p}\right)$ as the volume of the parallelepiped generated by the vectors $u_{1}, u_{2}, \cdots, u_{p}$. More precisely, we choose an orthonormal $p$-frame $\zeta=\left(w_{1}, w_{2}, \cdots, w_{p}\right), w_{i} \in T_{x} M, \quad i=1, \cdots, p$, which generates the same linear subspace of $T_{x} M$ as $\xi$ does and take a unique $p \times p$ matrix $A$ with $\xi=\zeta A$. Then we define the volume of $\xi$ by

$$
\operatorname{vol}(\xi):=|\operatorname{det} A|
$$

We remark that the volume $\operatorname{vol}(\xi)$ does not depend on the choice of $\zeta$, since the determinate of a transition matrix between two orthonormal frames is \pm 1 . Hence, 
$\operatorname{vol}(\xi)$ is well defined. Given any linear surjective transformation $\Phi$ from one Euclid space $E$ to another Euclid space $F$, we define

$$
\operatorname{det}(\Phi)=\frac{\operatorname{vol}(\Phi(\xi))}{\operatorname{vol}(\xi)}
$$

where $\xi$ is a frame spanning $E$. The definition does not depend on the choice of $\xi$.

Let $f$ be a $C^{1}$ diffeomorphism of $M$. For $1 \leq i \leq k$, we define functions $\phi_{i}, \psi_{i}$ $(1 \leq i \leq k)$ on $\mathcal{W}\left(l_{1}, \cdots, l_{k}\right)$ :

$$
\begin{aligned}
& \phi_{i}(\gamma)=\log \left|\operatorname{det}\left(\left.D f_{x}\right|_{F_{i}}\right)\right|, \\
& \psi_{i}(\gamma)=\log \left|\operatorname{det}\left(\left.D f_{x}^{-1}\right|_{H_{i}}\right)\right|=-\log \left|\operatorname{det}\left(\left.\left(D f_{f^{-1} x}\right)\right|_{\left(D f_{f^{-1} x_{x}}\right)^{-1} H_{i}}\right)\right|,
\end{aligned}
$$

where $\gamma=\left(F_{1}, \cdots, F_{k} ; H_{1}, \cdots, H_{k}\right) \in \mathcal{W}\left(l_{1}, \cdots, l_{k} ; x\right)$. Then $\phi_{i}, \psi_{i}(1 \leq i \leq k)$ are bounded and continuous on $\mathcal{W}\left(l_{1}, \cdots, l_{k}\right)$. For $1 \leq i \leq k$, we define projections

$$
\pi_{i}(\gamma)=F_{i}, \quad \widehat{\pi}_{i}(\gamma)=H_{i}
$$

In what follows, we suppose $f$ preserves an ergodic measure $\omega$. Then by the Oseledets Theorem [5] there exist

(a) real numbers $\lambda_{1}<\cdots<\lambda_{s}(s \leq d)$;

(b) positive integers $n_{1}, \cdots, n_{s}$, satisfying $n_{1}+\cdots+n_{s}=d$;

(c) a Borel set $O(\omega)$, called an Oseledets basin of $\omega$, satisfying $f(O(\omega))=O(\omega)$ and $\omega(O(\omega))=1$

(d) a measurable splitting, called an Oseledets splitting,

$$
T_{x} M=E_{1}(x) \oplus \cdots \oplus E_{s}(x)
$$

with $\operatorname{dim} E_{i}(x)=n_{i}$ and $D f\left(E_{i}(x)\right)=E_{i}(f x)$,

such that

$$
\lim _{n \rightarrow \pm \infty} \frac{\log \left\|D f^{n} v\right\|}{n}=\lambda_{i}
$$

for $\forall x \in O(\omega), v \in E_{i}(x) \backslash\{0\}, i=1,2, \cdots, s$.

(a) and (d) above allow us to arrange the Oseledets splitting according to the increasing order of the Lyapunov exponents. To avoid excessive terminology, we will arrange the Oseledets splitting at every point in the Oseledets basin in this way throughout this paper without explanation. We call the measure $\omega$ hyperbolic if none of its Lyapunov exponents is zero.

From now on, suppose $f: M \rightarrow M$ is a $C^{1+r}$ diffeomorphism, i.e., the derivative $D f$ is $r$-Hölder continuous. We give a quick review concerning some notions and results of Pesin theory. We point the reader to [3], [8, [7] for more details.

Definition 1.1. Given $b_{1}, b_{2} \gg \epsilon>0$, and for all $k \in \mathbb{Z}^{+}$, the Pesin block $\Lambda_{k}=$ $\Lambda_{k}\left(b_{1}, b_{2} ; \epsilon\right)$ consists of all points $x \in M$ for which there is a splitting $T_{x} M=$ $E_{x}^{s} \oplus E_{x}^{u}$ with the invariance property $\left(D f^{m}\right) E_{x}^{s}=E_{f^{m} x}^{s}$ and $\left(D f^{m}\right) E_{x}^{u}=E_{f^{m} x}^{u}$, and satisfying:

(a) $\left\|D f^{n} \mid E_{f^{m} x}^{s}\right\| \leq e^{\epsilon k} e^{-\left(b_{2}-\epsilon\right) n} e^{\epsilon|m|}, \forall m \in \mathbb{Z}, n \geq 1$;

(b) $\left\|D f^{-n} \mid E_{f^{m} x}^{u}\right\| \leq e^{\epsilon k} e^{-\left(b_{1}-\epsilon\right) n} e^{\epsilon|m|}, \forall m \in \mathbb{Z}, n \geq 1$; and

(c) $\tan \angle\left(E_{f^{m} x}^{s}, E_{f^{m} x}^{u}\right) \geq e^{-\epsilon k} e^{-\epsilon|m|}, \forall m \in \mathbb{Z}$.

Definition 1.2. $\Lambda\left(b_{1}, b_{2} ; \epsilon\right)=\bigcup_{k=1}^{+\infty} \Lambda_{k}\left(b_{1}, b_{2} ; \epsilon\right)$ is a Pesin set. 
We say an invariant measure $\mu$ is associated with the Pesin set $\Lambda=\Lambda\left(b_{1}, b_{2} ; \epsilon\right)$ if $\mu\left(\Lambda\left(b_{1}, b_{2} ; \epsilon\right)\right)=1$. Take and fix a hyperbolic ergodic measure $\omega$. Let $b_{1}, b_{2}$ be small such that $\omega$ has no Lyapunov exponents in $\left[-b_{2}, b_{1}\right]$; then $\omega\left(\Lambda\left(b_{1}, b_{2} ; \epsilon\right)\right)=1$.

Definition 1.3. Let $E$ and $F$ be two $D f$-invariant sub-bundles of $T M$. The angle between $E(x)$ and $F(x), x \in M$, is defined as follows:

$$
\sin \angle(E(x), F(x)):=\inf _{0 \neq u \in E(x), 0 \neq v \in F(x)} \sin \angle(u, v)=\inf _{0 \neq u \in E(x), 0 \neq v \in F(x)} \frac{\|u \wedge v\|}{\|u\|\|v\|},
$$

where $\wedge$ denotes the wedge product. For almost all $x \in M$, the limit

$$
\lim _{n \rightarrow+\infty} \frac{1}{n} \sum_{i=0}^{n-1} \angle\left(D f^{i} E(x), D f^{i} F(x)\right)
$$

exists. We call it the mean angle between $E$ and $F$ at $x$ and denote the limit by $m \angle(E(x), F(x))$.

This definition applies to the case of zero-dimensional intersection. We need the following notion to discuss the angle between two invariant bundles with higher dimensions.

Given $v \in \mathbb{R}^{d}$ and a linear subspace $E \subset \mathbb{R}^{d}$, define

$$
\operatorname{Proj}(v, E)= \begin{cases}\text { the orthogonal projection of } v /\|v\| \text { onto } E, & v \neq 0 \\ 0, & v=0 .\end{cases}
$$

We adopt the classic metric $d_{G}$ on the Grassmann bundle $\bigcup_{0 \leq i \leq d} G(i)$ :

$$
d_{G}\left(F, F^{\prime}\right)=\sup _{v \in \mathbb{R}^{d}}\left\|\operatorname{Proj}(v, F)-\operatorname{Proj}\left(v, F^{\prime}\right)\right\|,
$$

where $F, F^{\prime} \in \bigcup_{0 \leq i \leq d} G(i)$. When $F, F^{\prime}$ are disjoint except for the origin, $d_{G}$ is equivalent to the angle $\angle$. Note that $M$ can be embedded into some Euclid space $\mathbb{R}^{q}$, so $d_{G}$ induces a metric on the Grassmann bundle $\bigcup_{x \in M} \bigcup_{0 \leq i \leq d} G(x, i)$ as follows:

$$
d_{G}\left(F(x), F^{\prime}(y)\right)=d(x, y)+d_{G}\left(\bar{F}(x), \overline{F^{\prime}}(y)\right),
$$

where $F(x) \in \bigcup_{0 \leq i \leq d} G(x, i), F^{\prime}(y) \in \bigcup_{0 \leq i \leq d} G(y, i)$ and $\bar{F}(z) \ni 0$ is the translation of $F(z)$ in $\mathbb{R}^{\bar{q}}$.

Definition 1.4. Let $E$ and $F$ be two $D f$-invariant sub-bundles of $T_{x} M$. The limit

$$
\lim _{n \rightarrow+\infty} \frac{1}{n} \sum_{i=0}^{n-1} d_{G}\left(D f^{i} E(x), D f^{i} F(x)\right)
$$

exists for almost every $x \in M$. We call the limit the mean distance between $E$ and $F$ at $x$ and denote it by $m d_{G}(E(x), F(x))$.

Recall that $f$ preserves an ergodic measure $\omega$ with its Oseledets splitting

$$
T_{x} M=E_{1}(x) \oplus \cdots \oplus E_{s}(x), \quad s \leq d=\operatorname{dim} M, \quad x \in O(\omega) .
$$

By the Birkhoff Ergodic Theorem, there is an $\omega$-full measure subset in $O(\omega)$ such that every point $x$ in this subset satisfies

$$
m d_{G}\left(E_{i}(x), E_{j}(x)\right)=\int d_{G}\left(E_{i}(y), E_{j}(y)\right) d \omega(y) .
$$

Since different subspaces tangent at the same point $x$ in the Oseledets splitting intersect only at the origin, $d_{G}\left(E_{i}(x), E_{j}(x)\right)$ coincides with the angle between 
$E_{i}(x)$ and $E_{j}(x)$. Thus we call $\int d_{G}\left(E_{i}(y), E_{j}(y)\right) d \omega(y)$ the mean angle between $E_{i}:=\bigcup_{x \in O(\omega)} E_{i}(x)$ and $E_{j}:=\bigcup_{x \in O(\omega)} E_{j}(x)$ and write it as $m d_{G}^{\omega}\left(E_{i}, E_{j}\right), \forall 1 \leq$ $i \neq j \leq s$.

We call the flag of sub-bundles

$$
\begin{gathered}
\left(E_{1}, E_{1} \oplus E_{2}, \cdots, \bigoplus_{i=1}^{s-1} E_{i} ; \bigoplus_{i=2}^{s} E_{i}, \cdots, E_{s-1} \bigoplus E_{s}, E_{s}\right) \\
=\bigcup_{x \in O(\omega)} \chi\left(\left(E_{1}(x), \cdots, E_{s}(x)\right)\right)
\end{gathered}
$$

the Oseledets flag.

Given $\gamma=\left(F_{1}, \cdots, F_{t}\right)$ with $F_{j} \in \bigcup_{0 \leq i \leq d} G(i, x)(1 \leq j \leq t)$, denote by $A(\gamma)$ the matrix

$$
\left(\cos d_{G}\left(F_{i}, F_{j}\right)\right)_{t \times t}
$$

Let $\sigma(\gamma)$ denote the set of all eigenvalues of $A(\gamma)$ and let $\tau(\gamma)$ be the smallest eigenvalue. We call $\tau(\gamma)$ the independence number of $\gamma$. Note that $A(\gamma)$ is a real positive-definite symmetric matrix; therefore, $\sigma(\gamma) \subset(0,+\infty)$.

For $x \in O(\omega)$, we define the independence number of $x$ by the independence number of bundles at $x$ whose elements are all on different Oseledets bundles. More precisely, take $\gamma(x)=\left(E_{1}(x), E_{2}(x), \cdots, E_{s}(x)\right)$. Then we define

$$
\tau(x):=\tau(\gamma),
$$

the smallest eigenvalue of $A(\gamma)$. Clearly, $\tau(x)$ is well defined for $x \in O(\omega)$. Moreover, by the Birkhoff Ergodic Theorem, the equation

$$
\lim _{n \rightarrow+\infty} \frac{1}{n} \sum_{i=0}^{n-1} \tau\left(f^{i} x\right)=\int \tau(y) d \omega(y)
$$

holds on an $\omega$-full measure subset of $O(\omega)$. Therefore, we can define the independence number of $\omega$ by

$$
\tilde{\tau}(\omega):=\int \tau(y) d \omega(y)
$$

The following proposition is about the continuity of $\tau$. Note that this result is about the continuity of the smallest eigenvalue of matrices, analogous to Proposition 2.6 in [4], and hence we will give the proof in the appendix.

Proposition 1.5. Given a positive number $s \in \mathbb{Z}$ and a column of integers $n_{1}, n_{2}$, $\cdots, n_{s}$ with $1 \leq n_{i} \leq d$ and $\sum_{i=1}^{s} n_{i}=d$, the function $\tau: \mathcal{V}\left(n_{1}, n_{2}, \cdots, n_{s}\right) \rightarrow \mathbb{R}$ is continuous.

Let $\gamma_{1}=\left(E_{1}, E_{2}, \cdots, E_{s}\right)$ and $\gamma_{2}=\left(F_{1}, F_{2}, \cdots, F_{s}\right)$ be two elements in the bundle $\mathcal{V}\left(n_{1}, n_{2}, \cdots, n_{s}\right)$. We define the distance between $\gamma_{1}$ and $\gamma_{2}$ by

$$
d_{G}\left(\gamma_{1}, \gamma_{2}\right):=\max _{1 \leq i \leq s} d_{G}\left(E_{i}, F_{i}\right)
$$

Recall that $f$ preserves an ergodic measure $\omega$ with its Oseledets splitting

$$
T_{x} M=E_{1}(x) \oplus \cdots \oplus E_{s}(x), \quad s \leq d=\operatorname{dim} M, \quad x \in O(\omega),
$$

arranged according to the increasing order of Lyapunov exponents.

Suppose there is another ergodic hyperbolic measure $\omega^{\prime}$ with a $D f$-invariant (both forward and backward invariant) splitting

(4) $T_{y} M=E_{1}(y) \oplus E_{2}(y) \oplus \cdots \oplus E_{s}(y), \quad \operatorname{dim}\left(E_{j}\right)=n_{j}, \quad 1 \leq j \leq s, \quad y \in O\left(\omega^{\prime}\right)$. 
In order to correspond to the Oseledets splitting of $\omega$, throughout this note we only consider the invariant splittings which are arranged according to the increasing order of Lyapunov exponents, i.e., for any nonzero vectors $v \in E_{i}(y), w \in E_{j}(y)$, $1 \leq i<j \leq s$, we have

$$
\limsup _{n \rightarrow \infty} \frac{1}{n} \log \left\|D f^{n} v\right\|<\liminf _{n \rightarrow \infty} \frac{1}{n} \log \left\|D f^{n} w\right\| .
$$

In this setting, each $E_{j}(y)(1 \leq j \leq s)$ may not be an Oseledets subspace but is necessarily constituted by some adjacent Oseledets subspaces.

Definition 1.6. Let $\eta>0$. The Oseledets splitting (3) of $\omega$ is $\eta$ approximated by (4) of $\omega^{\prime}$, if there exists a measurable subset $\Gamma$ satisfying:

(a) $\omega(\Gamma)>1-\eta$;

(b) for any $x \in \Gamma$, there exist a point $z=z(x) \in \operatorname{supp}\left(\omega^{\prime}\right) \cap O\left(\omega^{\prime}\right)$ and $\varsigma(z)=$ $\left(E_{1}(z), \cdots, E_{s}(z)\right) \in \mathcal{V}\left(n_{1}, \cdots, n_{s} ; z\right)$ such that

$$
d_{G}(\gamma, \varsigma)<\eta
$$

where $\gamma(x)=\left(E_{1}(x), \cdots, E_{s}(x)\right) \in \mathcal{V}\left(n_{1}, \cdots, n_{s} ; x\right)$.

Remark 1.7. Here the loss of small measure $1-\omega(\Gamma)$ is necessary, since we cannot ensure in general that the approximation property holds on a full-measured set. Take the Lorenz attractor as an example. There are many periodic points arbitrarily close to its singularity in the measure theoretic sense. Let $\omega^{\prime}$ be the atomic measure on the singularity and $\omega$ be a periodic measure $\eta$-close to $\omega^{\prime}$. Then we find that only some of the points on the periodic orbit can be approximated by the singularity, the same as their Oseledets splittings.

Remark 1.8. Note that some subspace $E_{i}(z)$ in $\varsigma(z)$ may be a direct sum of adjacent Oseledets subspaces at $z$. Hence, the Oseledets splitting of $\omega$ is $\eta$-approximated by that of $\omega^{\prime}$, but not vice versa. In other words, $\eta$ is not a 'distance' between the Oseledets splitting of $\omega$ and that of $\omega^{\prime}$. This approximation is transitive but not symmetric. This is also the reason that we fix the dimensions of subspaces in Proposition 1.5.

Remark 1.9. We can do some modification in Definition [1.6 such that $\eta$ is defined transitively and symmetric, i.e., a distance. by

Given $\eta>0$, define the $\eta$-bundle distance of $\omega, \omega^{\prime}$, with respect to $\left(n_{1}, \cdots, n_{s}\right)$,

$$
d_{\eta}\left(\omega, \omega^{\prime}\right)=\inf _{\omega(\Gamma)>1-\eta, \omega^{\prime}\left(\Gamma^{\prime}\right)>1-\eta} d_{H}\left(\Gamma_{\omega}, \Gamma_{\omega^{\prime}}^{\prime}\right)
$$

where

$$
\begin{aligned}
\Gamma_{\omega} & =\left\{\gamma(x)=\left(E_{1}(x), \cdots, E_{s}(x)\right) \in \mathcal{V}\left(n_{1}, \cdots, n_{s} ; x\right) \quad \text { as (13) } \mid x \in \Gamma\right\}, \\
\Gamma_{\omega^{\prime}}^{\prime} & =\left\{\varsigma(x)=\left(E_{1}(x), \cdots, E_{s}(x)\right) \in \mathcal{V}\left(n_{1}, \cdots, n_{s} ; x\right) \quad \text { as (44) } \mid x \in \Gamma^{\prime}\right\}, \\
d_{H}\left(\Gamma_{\omega}, \Gamma_{\omega^{\prime}}^{\prime}\right) & =\max \left\{\sup _{\gamma \in \Gamma_{\omega}} \inf _{\varsigma \in \Gamma_{\omega^{\prime}}^{\prime}} d_{G}(\gamma, \varsigma), \sup _{\varsigma \in \Gamma_{\omega^{\prime}}^{\prime}} \inf _{\gamma \in \Gamma_{\omega}} d_{G}(\gamma, \varsigma)\right\} .
\end{aligned}
$$

The Oseledets splitting (3) of $\omega$ is $\eta$ approximated by (4) of $\omega^{\prime}$ if there exists $\eta$ satisfying:

$$
d_{\eta}\left(\omega, \omega^{\prime}\right)<\eta
$$


It may be worth pointing out that while $d_{\eta}$ is symmetric for each $\eta$ and each $\left(n_{1}, \cdots, n_{s}\right)$ in Definition 1.6. we fix a $\left(n_{1}, \cdots, n_{s}\right)$ that depends on $\omega$, but not $\omega^{\prime}$, and by choosing a different $\left(n_{1}, \cdots, n_{s}\right)$, the distance may increase.

Now we state our main result of this paper.

Theorem 1.10. Let $f: M \rightarrow M$ be a $C^{1+r}$ diffeomorphism preserving an ergodic hyperbolic measure $\omega$ with its Oseledets splitting

$$
T_{\Lambda} M=E_{1}(\Lambda) \oplus E_{2}(\Lambda) \oplus \cdots \oplus E_{s}(\Lambda), \quad \operatorname{dim}\left(E_{i}\right)=n_{i}, \quad 1 \leq i \leq s,
$$

arranged according to the increasing order of Lyapunov exponents of $\omega$, where $\Lambda=$ $\bigcup_{k \geq 1} \Lambda_{k}$ is the Pesin set associated with $\omega$. Given $\varepsilon>0$, there is a hyperbolic periodic orbit $\operatorname{orb}(z, f)$ of period $p=p(\varepsilon)$ together with a $D f$-invariant splitting

$T_{f^{j} z} M=E_{1}\left(f^{j} z\right) \oplus E_{2}\left(f^{j} z\right) \oplus \cdots \oplus E_{s}\left(f^{j} z\right), \quad \operatorname{dim}\left(E_{i}\right)=n_{i}, \quad 1 \leq i \leq s, 1 \leq j \leq p$, at $f^{j} z$ arranged according to the increasing order of Lyapunov exponents of the orbit $\operatorname{orb}(z)$ such that the atomic measure $\omega_{z}$ supported on $\operatorname{orb}(z, f)$ is $\varepsilon$-close to $\omega$ in the weak ${ }^{*}$-topology and satisfies the following properties:

(i) The mean angle of $\omega$ and of $\omega_{z}$ are $\varepsilon$-close, that is,

$\left|m d_{G}^{\omega}\left(E_{i}(\Lambda), E_{j}(\Lambda)\right)-m d_{G}^{\omega_{z}}\left(E_{i}(\operatorname{orb}(z)), E_{j}(\operatorname{orb}(z))\right)\right|<\varepsilon, \quad \forall 1 \leq i \neq j \leq s$.

(ii) The independence numbers of $\omega$ and of $\omega_{z}$ are $\varepsilon-$ close, that is,

$$
\left|\tilde{\tau}(\omega)-\tilde{\tau}\left(\omega_{z}\right)\right|<\varepsilon .
$$

(iii) The Oseledets splitting of $\omega$ is $\varepsilon$-approximated by that of $\omega_{z}$.

This result discusses the same approximation properties as stated in Theorem 1.12 of [4, but without the hypotheses of simple spectrum.

\section{Proof of the theorem}

In the beginning, we will find an isolated ergodic invariant measure on $\mathcal{W}$ covering $\omega$, which is needed in the sequel. Denote by $\pi$ the projection of $\mathcal{W}$ to the state space $M$. For any $\gamma=\left(F_{1}, \cdots, F_{k} ; H_{1}, \cdots, H_{k}\right) \in \mathcal{W}(x)$, define

$$
D f \gamma=\left(D f_{x} F_{1}, \cdots, D f_{x} F_{k} ; D f_{x} H_{1}, \cdots, D f_{x} H_{k}\right) .
$$

Given a homeomorphism $T: X \rightarrow X$ of a compact metric space $X$, and an ergodic measure $m$ preserved by $T$, define the basin of $m$ by

$$
Q_{m}(X, T)=\left\{x \in X \mid \lim _{n \rightarrow \pm \infty} \frac{1}{n} \sum_{i=0}^{n-1} \phi\left(T^{i} x\right)=\int_{X} \phi d m, \forall \phi \in C^{0}(X, \mathbb{R})\right\} .
$$

By the Birkhoff Ergodic Theorem, $m\left(Q_{m}(X, T)\right)=1$.

Lemma 2.1. There exists one and only one invariant measure $\tilde{\omega} \in \mathcal{M}_{\text {inv }}(\mathcal{W}, D f)$ such that $\pi_{*}(\tilde{\omega})=\omega$ and

$$
\int_{\mathcal{W}} \phi_{i} d \tilde{\omega}=\sum_{j=1}^{i} n_{j} \lambda_{j}, \quad \int_{\mathcal{W}} \psi_{i} d \tilde{\omega}=-\sum_{j=i+1}^{s} n_{j} \lambda_{j}, \quad 1 \leq i \leq s-1,
$$

where $\phi_{i}, \psi_{i}$ are defined in (1). In addition, $\tilde{\omega}$ is ergodic and $\tilde{\omega}\left(\mathcal{W}_{1}\right)=1$. 
Proof. Take and fix a point $x \in O(\omega)$. Let $\gamma_{0}=\left(E_{1}(x), \cdots, E_{s}(x)\right) \in \mathcal{V}$ so that

$$
\begin{aligned}
\lim _{n \rightarrow+\infty} \frac{1}{n} \sum_{j=0}^{n-1} \phi_{i}\left(D f^{j} \gamma_{1}\right) & =\sum_{t=1}^{i} n_{t} \lambda_{t}, \quad 1 \leq i \leq s-1, \\
\lim _{n \rightarrow+\infty} \frac{1}{n} \sum_{j=0}^{n-1} \psi_{i}\left(D f^{j} \gamma_{1}\right) & =-\sum_{t=i+1}^{s} n_{t} \lambda_{t}, \quad 1 \leq i \leq s-1,
\end{aligned}
$$

where $\gamma_{1}=\chi\left(\gamma_{0}\right) \in \mathcal{W}$. Define a sequence of measures $\mu_{n}$ on $\mathcal{W}$ by

$$
\int \phi d \mu_{n}:=\frac{1}{n} \sum_{j=0}^{n-1} \phi\left(D f^{j} \gamma_{1}\right), \quad \forall \phi \in C^{0}(\mathcal{W}, \mathbb{R}) .
$$

By taking a subsequence when necessary we can assume that $\mu_{n} \rightarrow \nu_{0}$. It is standard to verify that $\nu_{0}$ is a $D f$-invariant measure and $\nu_{0}$ covers $\omega$, i.e., $\pi_{*}\left(\nu_{0}\right)=\omega$. Set

$$
Q(\mathcal{W}, D f):=\bigcup_{\nu \in \mathcal{M}_{\text {erg }}(\mathcal{W}, D f)} Q_{\nu}(\mathcal{W}, D f)
$$

Then $Q(\mathcal{W}, D f)$ is a $D f$-invariant total measure subset in $\mathcal{W}$. We have

$$
\begin{aligned}
& \omega\left(Q_{\omega}(M, f) \cap \pi Q(\mathcal{W}, D f)\right) \\
\geq & \nu_{0}\left(\pi^{-1} Q_{\omega}(M, f) \cap Q(\mathcal{W}, D f)\right) \\
= & 1 .
\end{aligned}
$$

Then the set

$$
\begin{aligned}
\mathcal{A}:=\left\{\mu \in \mathcal{M}_{\text {erg }}(\mathcal{W}, D f) \mid\right. & \exists \gamma \in Q(\mathcal{W}, D f), \pi(\gamma) \in Q_{\omega}(M, f), \text { s.t. } \\
\lim _{n \rightarrow \pm \infty} \frac{1}{n} \sum_{i=0}^{n-1} \phi\left(D f^{i} \gamma\right) & \left.=\int_{\mathcal{W}} \phi d \mu, \forall \phi \in C^{0}(\mathcal{W}, \mathbb{R})\right\}
\end{aligned}
$$

is nonempty. It is clear that $\mu$ covers $\omega, \pi_{*}(\mu)=\omega$, for all $\mu \in \mathcal{A}$; and we claim that $\mathcal{A}$ coincides with the set of all the measures in $\mathcal{M}_{\text {erg }}(\mathcal{W}, D f)$ that cover $\omega$. In fact, if $\mu \in \mathcal{M}_{\text {erg }}(\mathcal{W}, D f)$ covers $\omega, \pi_{*} \mu=\omega$, from the fact that $\mu\left(Q_{\mu}(\mathcal{W}, D f)\right)=1$, we have

$$
\begin{aligned}
& \omega\left(Q_{\omega}(M, f) \cap \pi Q_{\mu}(\mathcal{W}, D f)\right) \\
\geq & \mu\left(\pi^{-1} Q_{\omega}(M, f) \cap Q_{\mu}(\mathcal{W}, D f)\right) \\
= & 1 .
\end{aligned}
$$

Thus there is $\gamma^{\prime} \in Q_{\mu}(\mathcal{W}, D f)$ with $\pi\left(\gamma^{\prime}\right) \in Q_{\omega}(M, f)$, which means $\mu \in \mathcal{A}$. Therefore,

$$
\mathcal{A}=\left\{\mu \in \mathcal{M}_{\text {erg }}(\mathcal{W}, D f) \mid \pi_{*}(\mu)=\omega\right\} .
$$

Assume the ergodic decomposition of $\nu_{0}$ is of the form

$$
\nu_{0}=\int_{\mathcal{M}_{\text {erg }}(\mathcal{W}, D f)} d \tau_{\nu_{0}}(m) .
$$


Then $\tau_{\nu_{0}}(\mathcal{A})=1$. Since $\mu_{n} \rightarrow \nu_{0}$ and $\phi_{i}, \psi_{i}(1 \leq i \leq s-1)$ are continuous,

$$
\begin{aligned}
\int_{\mathcal{W}} \phi_{i} d \nu_{0} & =\lim _{n \rightarrow+\infty} \int_{\mathcal{W}} \phi_{i} d \mu_{n}=\sum_{t=1}^{i} n_{t} \lambda_{t}, \\
\int_{\mathcal{W}} \psi_{i} d \nu_{0} & =\lim _{n \rightarrow+\infty} \int_{\mathcal{W}} \psi_{i} d \mu_{n}=-\sum_{t=i+1}^{s} n_{t} \lambda_{t} .
\end{aligned}
$$

Using the Ergodic Decomposition Theorem, we obtain

$$
\begin{aligned}
(5) \int_{\mathcal{M}_{\text {erg }}(\mathcal{W}, D f)} \int_{\mathcal{W}} \phi_{i} d m d \tau_{\nu_{0}}(m) & =\int_{\mathcal{W}} \phi_{i} d \nu_{0}=\sum_{t=1}^{i} n_{t} \lambda_{t}, \quad 1 \leq i \leq s-1, \\
(6) \int_{\mathcal{M}_{\text {erg }}(\mathcal{W}, D f)} \int_{\mathcal{W}} \psi_{i} d m d \tau_{\nu_{0}}(m) & =\int_{\mathcal{W}} \psi_{i} d \nu_{0}=-\sum_{t=i+1}^{s} n_{t} \lambda_{t}, \quad 1 \leq i \leq s-1 .
\end{aligned}
$$

Observe that for any $m \in \mathcal{A}$, for $m$-almost every $\gamma(x) \in \mathcal{W}$,

$$
\int_{\mathcal{W}} \phi_{i} d m=\lim _{n \rightarrow+\infty} \frac{1}{n} \sum_{j=0}^{n-1} \phi_{i}\left(D f^{j} \gamma\right) \geq \sum_{t=1}^{i} n_{t} \lambda_{t}
$$

because $\pi_{i}(\gamma(x))$ has dimension $n_{1}+\cdots+n_{i}$ and any vector in

$$
\pi_{i}(\gamma(x)) \backslash \bigoplus_{t=1}^{i} E_{t}(x)
$$

converges under $D f^{j}$ to an Oseledets subspace with a Lyapunov exponent larger than $\lambda_{i}$. The equality holds if and only if $\pi_{i}(\gamma(x))=\bigoplus_{t=1}^{i} E_{t}(x)$. Hence, by (5), for $\tau_{\nu_{0}}$-almost every $m \in \mathcal{A}$, for $m$-almost every $\gamma(x) \in \mathcal{W}$,

$$
\int_{\mathcal{W}} \phi_{i} d m=\lim _{n \rightarrow+\infty} \frac{1}{n} \sum_{j=0}^{n-1} \phi_{i}\left(D f^{j} \gamma\right)=\sum_{t=1}^{i} n_{t} \lambda_{t}, \quad 1 \leq i \leq s-1 .
$$

In the same manner, applying the above argument for $D f^{-1}$, for any $m \in \mathcal{A}$, $m$-almost every $\gamma(x) \in \mathcal{W}$,

$$
\int_{\mathcal{W}} \psi_{i} d m=\lim _{n \rightarrow+\infty} \frac{1}{n} \sum_{j=0}^{n-1} \psi_{i}\left(D f^{-j} \gamma\right) \geq-\sum_{t=i+1}^{s} n_{t} \lambda_{t}
$$

and the equality holds if and only if $\widehat{\pi}_{i}(\gamma(x))=\bigoplus_{t=i+1}^{s} E_{t}(x)$. It follows that for $\tau_{\nu_{0}}$-almost every $m \in \mathcal{A}$, for $m$-almost every $\gamma(x) \in \mathcal{W}$,

$$
\int_{\mathcal{W}} \psi_{i} d m=\lim _{n \rightarrow+\infty} \frac{1}{n} \sum_{j=0}^{n-1} \psi_{i}\left(D f^{-j} \gamma\right)=-\sum_{t=i+1}^{s} n_{t} \lambda_{t}, \quad 1 \leq i \leq s-1 .
$$

Additionally, if $\nu_{0}^{\prime} \in \mathcal{M}_{i n v}(\mathcal{W}, D f)$ satisfying the fact that $\pi_{*}\left(\nu_{0}^{\prime}\right)=\omega$ and

$$
\int_{\mathcal{W}} \phi_{i} d \nu_{0}^{\prime}=\sum_{j=1}^{i} n_{j} \lambda_{j}, \quad \int_{\mathcal{W}} \psi_{i} d \nu_{0}^{\prime}=-\sum_{j=i+1}^{s} n_{j} \lambda_{j}, \quad 1 \leq i \leq s-1,
$$


then by the above proof we can see that for $\nu_{0}^{\prime}$-almost every $\gamma(x) \in \mathcal{W}$,

$$
\pi_{i}(\gamma(x))=\bigoplus_{t=1}^{i} E_{t}(x) \text { and } \widehat{\pi}_{i}(\gamma(x))=\bigoplus_{t=i+1}^{s} E_{t}(x), \forall 1 \leq i \leq s-1 .
$$

So $\nu_{0}^{\prime}=\nu_{0}$. That is, $\nu_{0}$ is unique and ergodic and $\nu_{0}\left(\mathcal{W}_{1}\right)=1$. Let $\tilde{\omega}=\nu_{0}$.

Remark 2.2. Lemma 2.1 implies that there is a unique invariant measure on $\mathcal{W}_{1}=$ $\mathcal{W}_{1}\left(\ell_{1}, \ell_{2}, \cdots, \ell_{s}\right)$ satisfying the requirement. From the proof, we can also tell that the equations hold only on the Oseledets flag (if $\ell_{i}$ is equal to the dimension of the direct sum of multiple Oseledets spaces with bottom $i$ Lyapunov exponents for every $1 \leq i \leq s)$. That is to say, there is some correspondence between invariant splittings and invariant measures. We have discussed how to find an invariant measure supported on a given bundle to cover the given ergodic measure on the state space $M$ in Theorem 2.7 of [4]. Here, $\tilde{\omega}$ is the invariant measure on $\mathcal{W}_{1}$ corresponding to the Oseledets flag indeed.

Remark 2.3. In the proof of the above lemma, $\gamma_{0}$ can be a column of all the Oseledets subspaces, or a column of invariant subspaces, some of which are direct sums of adjacent Oseledets subspaces. For this kind of coarser invariant splitting, Lemma 2.1 is still true, since the hyperbolic structure remains. The main idea that any subspace that does not have one of the bottom (rep. top) $i$ (rep. $s-i$ ) Lyapunov exponents will be attracted to a subspace with an upper (rep. lower) Lyapunov exponent upon forward iteration is still applicable. The only difference is that we should talk about another bundle $\mathcal{W}$ whose elements have a decreasing number of subspaces and an increasing dimension of subspaces.

Before proceeding to the proof of Theorem 1.10, we cite a crucial result on measure and Lyapunov exponents approximation in the following, which is a combination of the main result in [10] and Theorem 1.8 in [4].

Theorem 2.4. Let $f: M \rightarrow M$ be a $C^{1+r}(r>0)$ diffeomorphism on a compact manifold preserving an ergodic hyperbolic measure $\omega$. Then there exists an $f$-invariant set $\tilde{\Lambda}$ of $\omega$-full measure such that for any number $\varepsilon>0$ and any measure $\mu$ in $\mathcal{M}_{\text {inv }}(\tilde{\Lambda})$, the set of all $f$-invariant measures supported by $\tilde{\Lambda}$, there is a hyperbolic periodic orbit satisfying the following:

(1) the atomic measure supported on this hyperbolic periodic orbit is $\varepsilon$-close to $\mu$ in the weak ${ }^{*}$-topology,

(2) the Lyapunov exponents of the hyperbolic periodic orbit are $\varepsilon$-approximating those of $\mu$ :

$$
\left|\lambda_{j}-\widetilde{\lambda}_{j}\right|<\varepsilon, \quad j=1, \cdots, d,
$$

where $\lambda_{1} \leq \cdots \leq \lambda_{d}$ are the Lyapunov exponents of $\mu$ and $\widetilde{\lambda}_{1} \leq \cdots \leq \widetilde{\lambda}_{d}$ are the Lyapunov exponents of the hyperbolic periodic orbit.

Proof. Let us sketch the proof here. Let $\Lambda=\bigcup_{\ell=1}^{\infty} \Lambda_{\ell}$ be the Pesin set associated with $\omega$. We denote by $\left.\omega\right|_{\Lambda_{\ell}}$ the conditional measure of $\omega$ on $\Lambda_{\ell}$. Set $\tilde{\Lambda}_{\ell}=\operatorname{supp}\left(\left.\omega\right|_{\Lambda_{\ell}}\right)$ and $\tilde{\Lambda}=\bigcup_{\ell=1}^{\infty} \tilde{\Lambda}_{\ell}$. Then $\tilde{\Lambda}$ will meet the conditions of our theorem (we can intersect $\tilde{\Lambda}$ with $\Lambda$ of Theorem 3.1 in [10], if necessary).

We can construct a pseudo-orbit enough to approximate $\mu$ with any pre-assigned scale $\varepsilon$ in measure sense and find a hyperbolic periodic point shadowing the pseudoorbit as in the proof of Theorem 1.8 in 4]. Hence condition (1) is satisfied. Note 
that the obtained periodic point for the main result in [10] is chosen by Katok's closing lemma (see [2]); we can directly cite the related proof here to verify that it also meets condition (2).

Proof of Theorem 1.10, Take a decreasing sequence $\left\{\varepsilon_{n}\right\}_{n=1}^{\infty}$ which approaches zero. We further assume that

$$
\varepsilon_{1}<\frac{1}{2} \min \left\{\left|\lambda_{i}-\lambda_{j}\right| \mid 1 \leq i \neq j \leq s, \varepsilon\right\} .
$$

For each $n$, applying Theorem 2.4 to the ergodic measure $\omega$, we can find a hyperbolic periodic point $z_{n}$ with period $p_{n}$ which satisfies the following properties:

(a) The atomic measure $\omega_{n}=\frac{1}{p_{n}} \sum_{i=0}^{p_{n}-1} \delta_{f^{i} z_{n}}$ is $\varepsilon_{n}$-close to the measure $\omega$ in the weak*-topology.

(b) Without loss of generality we assume that $\omega_{n}$ has an invariant splitting $E_{1}^{n} \oplus E_{2}^{n} \oplus \cdots \oplus E_{s}^{n}$ on $T_{\operatorname{orb}\left(z_{n}, f\right)} M$ such that $\operatorname{dim}\left(E_{i}^{n}\right)=n_{i}(1 \leq i \leq s)$, and for any $0 \neq v \in E_{i}^{n}\left(z_{n}\right)$, the Lyapunov exponents

$$
\lambda_{i}-\varepsilon_{n}<\liminf _{k \rightarrow \infty} \frac{1}{k} \log \left\|D f_{z_{n}}^{k} v\right\| \leq \limsup _{k \rightarrow \infty} \frac{1}{k} \log \left\|D f_{z_{n}}^{k} v\right\|<\lambda_{i}+\varepsilon_{n} .
$$

Remark 2.5. As we discussed in Remark 1.8, some subspace $E_{i}^{n}$ may actually be a direct sum of multiple Oseledets subspaces for $\omega_{n}$. That is, a single Oseledets subspace for $\omega$ with multiplicity at least 2 may split into multiple subspaces (with very similar Lyapunov exponents) under the approximation by $\omega_{n}$, and we group these subspaces together in order to approximate the splitting.

Let $\gamma_{n}(x)=\left(E_{1}^{n}(x), E_{2}^{n}(x), \cdots, E_{s}^{n}(x)\right)$ for $x \in \operatorname{orb}\left(z_{n}\right)$. Thus, there is an ergodic invariant measure $\tilde{\omega}_{n} \in \mathcal{M}_{\text {erg }}\left(\mathcal{W}_{1}, D f\right)$ such that $\pi_{*}\left(\tilde{\omega}_{n}\right)=\omega_{n}$ and

$$
\tilde{\omega}_{n}\left(\chi\left(\gamma_{n}\left(f^{t} z_{n}\right)\right)\right)=\frac{1}{p_{n}}, \quad 0 \leq t \leq p_{n}-1 .
$$

At most taking a subsequence, we suppose that $\tilde{\omega}_{n}$ converges to $\mu \in \mathcal{M}_{\text {inv }}(\mathcal{W}, D f)$. Since $\pi_{*}\left(\tilde{\omega}_{n}\right)=\omega_{n}$ and $\lim _{n \rightarrow+\infty} \omega_{n}=\omega$, we have $\pi_{*}(\mu)=\omega$. By (7), it is verified that

$$
\begin{aligned}
\sum_{j=1}^{i} n_{j}\left(\lambda_{j}-\varepsilon_{n}\right) & <\int_{\mathcal{W}} \phi_{i} d \tilde{\omega}_{n}<\sum_{j=1}^{i} n_{j}\left(\lambda_{j}+\varepsilon_{n}\right), \quad 1 \leq i \leq s-1, \\
\sum_{j=i+1}^{s} n_{j}\left(-\lambda_{j}-\varepsilon_{n}\right) & <\int_{\mathcal{W}} \psi_{i} d \tilde{\omega}_{n}<\sum_{j=i+1}^{s} n_{j}\left(-\lambda_{j}+\varepsilon_{n}\right), \quad 1 \leq i \leq s-1 .
\end{aligned}
$$

Letting $n \rightarrow \infty$, we deduce that

$$
\begin{aligned}
\int_{\mathcal{W}} \phi_{i} d \mu & =\sum_{j=1}^{i} n_{j} \lambda_{j}, \quad 1 \leq i \leq s-1, \\
\int_{\mathcal{W}} \psi_{i} d \mu & =-\sum_{j=i+1}^{s} n_{j} \lambda_{j}, \quad 1 \leq i \leq s-1 .
\end{aligned}
$$

By Lemma 2.1, it follows that $\mu=\tilde{\omega}$.

Denote $\rho_{0}=\chi_{*}^{-1}(\tilde{\omega}), \rho_{n}=\chi_{*}^{-1}\left(\tilde{\omega}_{n}\right)$. Then $\rho_{0}, \rho_{n} \in \mathcal{M}_{\text {erg }}(\mathcal{V}, D f)$ and $\rho_{n} \rightarrow \rho_{0}$ as $n \rightarrow+\infty$. 
Proof of (i). We need show that for any $1 \leq i \neq j \leq s$,

$$
\lim _{n \rightarrow \infty} \frac{1}{p_{n}} \sum_{k=0}^{p_{n}-1} d_{G}\left(E_{i}^{n}\left(f^{k}\left(z_{n}\right)\right), E_{j}^{n}\left(f^{k}\left(z_{n}\right)\right)\right)=m d_{G}^{\omega}\left(E_{i}, E_{j}\right),
$$

where $E_{1}^{n}(\operatorname{orb}(z)) \oplus \cdots \oplus E_{s}^{n}(\operatorname{orb}(z))$ is the invariant splitting given as in (b) above. We define $\phi_{i j}: \mathcal{U} \rightarrow \mathbb{R},\left(E_{1}, \cdots, E_{s}\right) \rightarrow d_{G}\left(E_{i}, E_{j}\right)$. Then $\phi_{i j}$ is bounded and continuous. Noting that $\rho_{n} \rightarrow \rho_{0}$, we get (8).

Proof of (ii). We recall the following relations:

$$
\omega_{n} \rightarrow \omega, \quad \rho_{n} \rightarrow \rho_{0}, \quad(\pi \circ \chi)_{*}\left(\rho_{n}\right)=\omega_{n}, \quad(\pi \circ \chi)_{*}\left(\rho_{0}\right)=\omega .
$$

Since the function $\tau: \mathcal{V} \rightarrow \mathbb{R}$ is continuous by Proposition 1.5, we have

$$
\int \tau d \rho_{n} \rightarrow \int \tau d \rho_{0}
$$

Moreover, note that the ergodic measure $\tilde{\omega} \in \mathcal{M}_{\text {erg }}(\mathcal{W}, D f)$ is unique in the sense of Lemma 2.1. By the discussion in Remark 2.2 and the definition of $\rho_{0}$, it holds that $\rho_{0}$ is supported on the bundle consisting of all Oseledets subspaces in order. Hence, we have that

$$
\int \tau d \rho_{0}=\int \tau d \omega
$$

Also note that $\omega_{n}$ is an ergodic measure whose spectra are increasingly arranged as $E_{1}^{n} \oplus \cdots \oplus E_{s}^{n}$. Though the splitting of $\omega_{n}$ may be coarser than the Oseledets splitting as discussed in Remark [2.5, it holds that

$$
\int \tau d \rho_{n}=\int \tau d \omega_{n}
$$

as talked about in Remark 2.3. Hence we have that $\int \tau d \omega_{n} \rightarrow \int \tau d \omega$ or $\tilde{\tau}\left(\omega_{n}\right) \rightarrow$ $\tilde{\tau}(\omega)$. We thus obtain (ii).

Proof of (iii). Given $\varepsilon>0$, take $l$ large so that

$$
\omega\left(\Lambda_{l}(\omega)\right)>1-\varepsilon,
$$

where $\Lambda_{l}(\omega)$ denotes the $l$-th Pesin block associated with $\omega$. Since the splitting

$$
x \rightarrow E_{1}(x) \oplus \cdots \oplus E_{s}(x)
$$

depends continuously on $x \in \Lambda_{l}(\omega)$, we can choose a uniform constant $\eta>0$ satisfying

$$
\eta<\min _{\substack{i \neq j \\ x \in \Lambda_{l}(\omega)}}\left\{\frac{1}{10} d_{G}\left(E_{i}(x), E_{j}(x)\right), \varepsilon\right\} .
$$

For each $x \in \Lambda_{l}(\omega) \cap \operatorname{supp}(\omega)$, we take and fix $\gamma_{0}(x)=\left(E_{1}(x), \cdots, E_{s}(x)\right)$. Denote by $B\left(\gamma_{0}(x), \eta\right)$ the $\eta$-neighborhood of $\gamma_{0}(x)$ under the metric on the Grassman bundle. Then $\rho_{0}\left(B\left(\gamma_{0}(x), \eta\right)\right)>0$. Recalling that $\rho_{n} \rightarrow \rho_{0}$, we have

$$
\liminf _{n \rightarrow+\infty} \rho_{n}\left(B\left(\gamma_{0}(x), \eta\right)\right) \geq \rho_{0}\left(B\left(\gamma_{0}(x), \eta\right)\right)>0 .
$$

Therefore, we can take an integer $N(x)=N\left(\gamma_{0}(x)\right)>0$ such that

$$
\rho_{n}\left(B\left(\gamma_{0}(x), \eta\right)\right)>0, \quad \forall n \geq N(x) .
$$


This implies the existence of an element $\beta_{n}(x)$ in $Q_{\rho_{n}}(\mathcal{U}, D f) \cap \operatorname{supp}\left(\rho_{n}\right) \cap B\left(\gamma_{0}(x), \eta\right)$ for each $n \geq N(x)$. Observing that $\rho_{n}$ covers the atomic measure $\omega_{n}$, we can deduce that $\beta_{n}(x)$ must be an element based on a periodic point $z(x, n) \in \operatorname{orb}\left(z_{n}\right)$, where $z_{n}$ is the periodic point chosen in $(a)$ and $(b)$. By the uniqueness of $\rho_{n}$, we know that $\beta_{n}(x)=\left(E_{1}^{n}(z(x, n)), \cdots, E_{s}^{n}(z(x, n))\right)$. Thus the Oseledets splitting of $\omega$ at $x$ is $\eta$ approximated by the invariant splitting $E_{1}^{n}(z(x, n)) \oplus \cdots \oplus E_{s}^{n}(z(x, n))$ of $\omega_{n}$ at $z(x, n), n \geq N(x)$. Note that the number $N(x)$ may vary with $x$; we need to find a number $N_{1}$, independent of the choice of $x \in \Lambda_{l}(\omega)$, such that $\rho_{N_{1}}$ meets (iii). This can be done by the compactness of $\Lambda_{l}(\omega)$ and continuity of the Oseledets splitting on it.

Hence we have completed the proof of Theorem 1.10

\section{Appendix}

Before proving Proposition 1.5. we need some lemmas. Denote by $\mathcal{L}\left(\mathbb{R}^{s}\right)$ the space of all linear maps on $\mathbb{R}^{s}$ and by $\sigma(L)$ the set of all eigenvalues of a linear map $L \in \mathcal{L}\left(\mathbb{R}^{s}\right)$. For any $\epsilon>0$, set

$$
\sigma_{\epsilon}(L):=\left\{\tau \in \mathbb{C} \mid \exists \tau^{\prime} \in \sigma(L) \text {, s.t. }\left|\tau-\tau^{\prime}\right|<\epsilon\right\} .
$$

Lemma 3.1 and Lemma 3.2 are cited from [6].

Lemma 3.1. For a given $L \in \mathcal{L}\left(\mathbb{R}^{s}\right)$ and $\epsilon>0$ there is $\delta>0$ such that $\sigma(T) \subset$ $\sigma_{\epsilon}(L)$ whenever $\|T-L\|<\delta$.

Lemma 3.2. Let $L \in \mathcal{L}\left(\mathbb{R}^{s}\right)$ and $\tau \in \sigma(L)$. There exist $\epsilon>0$ and $\delta>0$ with the property that for any $T \in \mathcal{L}\left(\mathbb{R}^{s}\right)$ with $\|T-L\|<\delta$ the cardinality of $\sigma(T) \cap B_{\epsilon}(\tau)$ is not more than the multiplicity of $\tau$, where $B_{\epsilon}(\tau)=\left\{\tau^{\prime} \in \mathbb{C}|| \tau-\tau^{\prime} \mid<\epsilon\right\}$.

Proof of Proposition 1.5. We assume by contrary that $\tau$ is not continuous at a frame $\alpha_{0} \in \mathcal{V}\left(n_{1}, n_{2}, \cdots, n_{s}\right)$. Then there must exist a constant $\epsilon_{0}>0$ such that for any $\delta>0$ we can find another frame $\beta=\beta(\delta) \in \mathcal{V}\left(n_{1}, n_{2}, \cdots, n_{s}\right)$ satisfying $d_{G}\left(\alpha_{0}, \beta\right)<\delta$ but $\left|\tau\left(\alpha_{0}\right)-\tau(\beta)\right|>2 \epsilon_{0}$. Arrange the eigenvalues of $A\left(\alpha_{0}\right)$ in decreasing order as $\left\{\tau_{1}>\tau_{2}>\cdots>\tau_{t}>\tau\left(\alpha_{0}\right)\right\}, t \leq s-1$. By Lemma 3.2, there are real numbers $\epsilon_{i}>0, \delta_{1}>0$ and $\delta^{\prime}>0$ such that for any $\gamma \in \mathcal{V}\left(n_{1}, n_{2}, \cdots, n_{s}\right)$ with $d\left(\gamma, \alpha_{0}\right)<\delta^{\prime}$, the following hold:

(a) the cardinality $\#\left[\sigma(\gamma) \cap B_{\epsilon_{i}}\left(\tau_{i}\right)\right]$ is not more than the multiplicity of $\tau_{i}$, and

(b) the cardinality $\#\left[\sigma(\gamma) \cap B_{\delta_{1}}\left(\tau\left(\alpha_{0}\right)\right)\right]$ is not more than the multiplicity of $\tau\left(\alpha_{0}\right)$, and

(c) $B_{\epsilon_{i}}\left(\tau_{i}\right) \cap B_{\delta_{1}}\left(\tau\left(\alpha_{0}\right)\right)=\emptyset, i=1, \cdots, t$.

Take

$$
0<\epsilon \leq \min \left\{\epsilon_{i}, \delta_{1} ; i=0,1, \cdots, t\right\} .
$$

Choose $\delta<\min \left\{\epsilon, \delta^{\prime}\right\}$ by Lemma 3.1 such that each $\gamma \in \mathcal{V}\left(n_{1}, n_{2}, \cdots, n_{s}\right)$ with $d\left(\gamma, \alpha_{0}\right)<\delta$ satisfies $\tau(\gamma) \in \bigcup_{i=1}^{t} B_{\epsilon}\left(\tau_{i}\right) \cup B_{\epsilon}\left(\tau\left(\alpha_{0}\right)\right)$. From the choice of $\epsilon_{0}$ and $\beta=\beta(\delta)$ we have $\tau(\beta) \in \bigcup_{i=1}^{t} B_{\epsilon_{i}}\left(\tau_{i}\right)$. Recall that $\tau(\beta)$ is the smallest eigenvalue of $A(\beta)$, and hence there is no eigenvalue of $A(\beta)$ inside $B_{\epsilon}\left(\tau\left(\alpha_{0}\right)\right)$. So we deduce 
by Lemma 3.1 and Lemma 3.2 that

$$
\begin{aligned}
s & =\# \sigma(\beta)=\#\left[\sigma(\beta) \cap B_{\epsilon}\left(\tau\left(\alpha_{0}\right)\right)\right]+\#\left[\sigma(\beta) \cap \bigcup_{i=1}^{t} B_{\epsilon}\left(\tau_{i}\right)\right] \\
& =\#\left[\sigma(\beta) \cap \bigcup_{i=1}^{t} B_{\epsilon}\left(\tau_{i}\right)\right] \\
& <\# \sigma\left(\alpha_{0}\right)=s,
\end{aligned}
$$

a contradiction. This implies that the function $\tau$ is continuous.

\section{ACKNOWLEDGEMENT}

The authors would like to thank the referee for helpful suggestions to improve the original manuscript.

\section{REFERENCES}

[1] Xiongping Dai, On the approximation of Lyapunov exponents and a question suggested by Anatole Katok, Nonlinearity 23 (2010), no. 3, 513-528, DOI 10.1088/0951-7715/23/3/004. MR:2586367 (2012c:37051)

[2] A. Katok, Lyapunov exponents, entropy and periodic orbits for diffeomorphisms, Inst. Hautes Études Sci. Publ. Math. 51 (1980), 137-173. MR573822 (81i:28022)

[3] A. Katok and L. Mendoza, Dynamical systems with non-uniformly hyperbolic behavior, supplementary chapter to Introduction to the modern theory of dynamical systems, by A. Katok and B. Hasselblatt, Cambridge University Press, 1995. MR.1326374 (96c:58055)

[4] Chao Liang, Geng Liu, and Wenxiang Sun, Approximation properties on invariant measure and Oseledec splitting in non-uniformly hyperbolic systems, Trans. Amer. Math. Soc. 361 (2009), no. 3, 1543-1579, DOI 10.1090/S0002-9947-08-04630-8. MR.2457408 (2009m:37085)

[5] V. I. Oseledec, A multiplicative ergodic theorem. Characteristic Ljapunov, exponents of dynamical systems (Russian), Trudy Moskov. Mat. Obšč. 19 (1968), 179-210. MR0240280 (39 \#1629)

[6] Jacob Palis Jr. and Welington de Melo, Geometric theory of dynamical systems, An introduction. Translated from the Portuguese by A. K. Manning. Springer-Verlag, New York, 1982. MR669541(84a:58004)

[7] Y. Pesin, Lyapunov characteristic exponents and ergodic properties of smooth dynamical systems with an invariant measure, Sov. Math. Dok. 17 (1976), 196-199.

[8] Mark Pollicott, Lectures on ergodic theory and Pesin theory on compact manifolds, London Mathematical Society Lecture Note Series, vol. 180, Cambridge University Press, Cambridge, 1993. MR:1215938 (94k:58080)

[9] Wenxiang Sun and Edson Vargas, Entropy and ergodic probability for differentiable dynamical systems and their bundle extensions, Topology Appl. 154 (2007), no. 3, 683-697, DOI 10.1016/j.topol.2006.09.006. MR2280913 (2008f:37052)

[10] Zhenqi Wang and Wenxiang Sun, Lyapunov exponents of hyperbolic measures and hyperbolic periodic orbits, Trans. Amer. Math. Soc. 362 (2010), no. 8, 4267-4282, DOI 10.1090/S00029947-10-04947-0. MR2608406 (2011m:37030)

School of Statistics and Mathematics, Central University of Finance and Economics, Beijing 100081, People's Republic of China

E-mail address: chaol@cufe.edu.cn

School of Mathematical Sciences, Peking University, Beijing 100871, People's RePUBLIC OF CHINA

E-mail address: liaogang@math.pku.edu.cn

LMam, School of Mathematical Sciences, Peking University, Beijing 100871, PeoPLE'S REPUBlic OF CHINA

E-mail address: sunwx@math.pku.edu.cn 